\title{
Performance evaluation of a compact 10-GHz pulse compressor based on a highly nonlinear Bismuth-Oxide fibre
}

\author{
S. Asimakis, T. T. Ng, M A.F. Roelens, P. Petropoulos, D. J. Richardson \\ Optoelectronics Research Centre, University of Southampton, Southampton, SO17 1BJ, UK \\ G. Meloni, A. Bogoni, L. Potí \\ Integrated Research Center for Photonic Networks and Technologies, Scuola Superiore Sant'Anna, Pisa, Italy
}

There are a variety of mode-locked lasers suitable for telecommunications applications capable of generating picosecond-long pulses at high repetition rates. Although the direct generation of even shorter pulses at such high rates is possible it is generally considered far easier to compress the pulses of mode-locked lasers when sub-picosecond pulses are required. However, this can lead to problems in terms of pulse synchronisation and stability as km scale lengths of dispersion shifted or highly nonlinear silica fibre are typically needed. In this paper, a pulse compressor operating at $10 \mathrm{GHz}$ is realized using just 2 meters of a connectorized Bismuth-Oxide highly-nonlinear fibre (Bi-HNLF). We further present the complete phase and intensity characterisation of the compressed pulses using a linear form of Frequency-Resolved Optical Gating (L-FROG) [1] based on a commercially available lithium niobate modulator.

The Bi-HNLF has an ultra-high nonlinearity $\gamma$ of $1100 \mathrm{~W}^{-1} \mathrm{~km}^{-1}$ and a normal dispersion of $-252 \mathrm{ps} / \mathrm{nm} / \mathrm{km} \mathrm{at}$ $1550 \mathrm{~nm}$. Its extreme nonlinearity allows significant spectral broadening to be achieved at reasonable power levels even at high pulse repetition rates. At the same time, the normal dispersion of the fibre leads to the formation of pulses with a linear chirp profile, which can easily be compressed by propagation through an appropriate length of anomalously dispersive fibre. In our experiments we amplified $10 \mathrm{GHz}, 2 \mathrm{ps} \mathrm{sech}{ }^{2}$ pulses to $\sim 25 \mathrm{dBm}$ and launched them into the BiHNLF. The pulse spectrum at the output of the fibre was then broadened to $\sim 35 \mathrm{~nm}$ at the 20dB level. Linear pulse compression was then performed in $31 \mathrm{~m}$ of SMF. We characterised the compressed pulses using L-FROG where the gating was performed either using an electro-absorption modulator (EAM) [1] or a $\mathrm{LiNbO}_{3}$ Mach-Zehnder modulator (MZM). L-FROG has the advantage of a higher sensitivity as compared to the more conventional second-harmonic generation FROG, however the pulse widths that can be reliably characterised are typically limited by the duration of the optical gate, or more crucially by the optical bandwidth of the gating devices. This is clearly shown in Fig.1a, which compares the directly measured spectrum on an optical spectrum analyser to the retrieved spectrum obtained both with EAM- and MZM-based L-FROG. The wavelength cut-off of the semiconductor EAM prohibits sampling of wavelength components below $\sim 1540 \mathrm{~nm}$, thus making this device inadequate for characterizing broadband pulses. The retrieved spectrum obtained from the MZM-based L-FROG measurement on the other hand, matches well the directly measured spectrum, owing to the much broader optical bandwidth of this modulator. The retrieved temporal pulse shape and chirp profile obtained from this measurement was compared to numerical simulations based on the split-step Fourier method, and good agreement was obtained (see Fig.1(b)). Both measurement and simulations indicated a pulse width of $\sim 400 \mathrm{fs}$, and a minimal chirp across the pulse, indicating that maximum pulse compression was achieved.

(a)

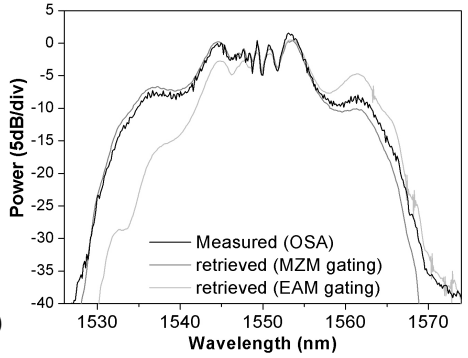

(b)

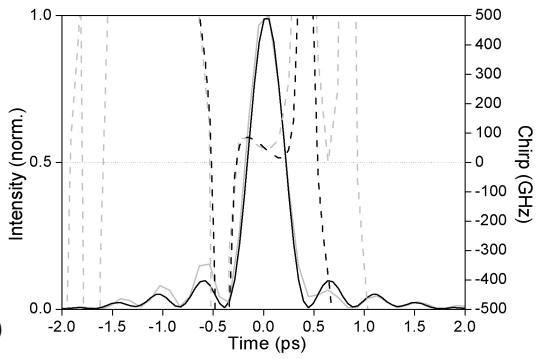

Fig. 1 (a) Directly measured and retrieved spectra of the compressed pulses after the Bi-HNLF, (b) Pulse shape (solid lines) and chirp profile (dashed lines) comparison between numerical simulations (black lines) and retrieved pulses (grey lines) using MZM-based L-FROG.

In conclusion, we have demonstrated 5-fold pulse compression at $10 \mathrm{GHz}$ using a fully-fiberised set-up based on a 2-metre long Bi-HNLF. The high nonlinearity and low two-photon absorption of the Bi-HNLF (as compared to other compound glass fibres [2]) allows the use of higher pump powers and therefore higher compression ratios to be achieved. Complete characterisation of the compressed pulses with high-sensitivity is reported using an L-FROG system based on commercial devices. These pulses are the shortest ever to be characterised using L-FROG.

The authors acknowledge Asahi Glass Company for the provision of the Bi-HNLF. This work was partially supported by EU NoE e-Photon/ONe IST-027497, VD-T and JP-T.

\section{References}

1. B. Thomsen et al., IEEE Photon. Tech. Lett. 17, 1914-1916 (2006).

2. L. Fu et al., Appl. Phys. Lett. 88, 081116 (2006). 